\title{
ESTRUTURA FLORAL DAS ANGIOSPERMAS USADAS POR HELICONIUS ERATO PHYLLIS (LEPIDOPTERA, NYMPHALIDAE) NO RIO GRANDE DO SUL, BRASIL ${ }^{1}$
}

\author{
Claudine A. Corrêa ${ }^{2}$ \\ Bruno E. Irgang ${ }^{3}$ \\ Gilson R.P. Moreira ${ }^{2}$
}

\begin{abstract}
FLOWER STRUCTURE OF ANGIOSPERMS USED BY HELICONIUS ERATO PHYLLIS (LEPIDOPTERA, NYMPHALIDAE) IN RIO GRANDE DO SUL STATE, BRAZIL. A field survey of flowering plants used as food resource by the adults of Heliconius erato phyllis (Fabricius, 1775) was carried out in four sites located in Rio Grande do Sul state, Brazil. Samples were taken in preserved areas of the Atlantic Rain and Myrtaceae forests, an Eucalyptus plantation, and an urban park. Adult feeding frequencies on flowers were registered monthly from December 1996 to May 1997, on plants located on previously marked $200 \mathrm{~m}$ long transects. Flowers on which $H$. erato phyllis fed in the field were collected, drawn and morphometrically characterized. Feeding was registered on flowers of twenty-three species, of which seventeen are new records for $H$. erato in Brazil . The use of a given plant varied among localities, as a function of its corresponding abundance. The most visited flowers were those of Lantana camara L. and Stachytarpheta cayennensis (Rich.) Vahl, followed by Dahlia pinnata Voss in the urban site. The data suggest the existence of size and shape convergence between the proboscis and the small, tubular flowers upon which $H$. erato phyllis feeds. They also indicate that $H$. erato phyllis adults have an opportunistic nectar feeding / pollen gathering habit, using several of those flowers available in a given time and locality that fit such a morphometrical pattern. Since plant species of both primitive and derived families are used, there is no indication that phylogenetic constraints play a major role in this association, nor that color of flowers, growth pattern or size of the plants are relevant in determining their use by H. erato phyllis.
\end{abstract}

KEYWORDS. Pollination, pollen feeding, Lepidoptera, ecology, Heliconius erato phyllis.

\section{INTRODUCÃO}

As variadas relações mutualísticas entre animais e plantas há tempo despertam a atenção dos naturalistas (DARWIN, 1877), especialmente pelo elevado número de requintados mecanismos envolvidos, como a polinização. Tal afirmativa é particularmente verdadeira dadas as diversas adaptações recíprocas existentes entre plantas e polinizadores (FAegri \& PiJl, 1971; CREPET, 1983; Bertin, 1989; BARTH, 1991; Herrera,

1. Contribuição Departamento Zoologia / UFRGS, nº. 363.

2. Departamento de Zoologia, Instituto de Biociências, UFRGS, Av. Paulo Gama, 40, 90046-900, Porto Alegre, RS, Brasil.

3. Departamento de Botânica, Instituto de Biociências, UFRGS, Av. Paulo Gama, 40, 90046-900, Porto Alegre, RS, Brasil. 
1996). Neste contexto, situam-se as síndromes florais propostas porFAEGRI \& PIJL (1971); segundo esta teoria, as flores apresentam um conjunto de características que as condicionam a serem utilizadas com maior freqüência por um ou outro agente polinizador em potencial. Os lepidópteros enquadram-se em duas síndromes: psicofilia e falenofilia. As flores ditas psicófilas são aquelas utilizadas por borboletas e caracterizam-se pela atividade diurna, pelo odor leve e agradável e comumente pelas cores vivas. Geralmente são eretas, de simetria radial e com a borda da corola grande, o que permite o pouso das borboletas. É comum apresentarem nectários grandes contidos em estruturas tubiformes ou esporões florais. Já as flores falenofílicas, utilizadas pelas mariposas, em sua grande maioria são de atividade noturna, de odor mais marcante, geralmente de cores pálidas e, de modo geral, suas corolas não têm bordas proeminentes. As diferentes características encontradas nesses dois tipos de flores relacionam-se diretamente ao hábito do inseto, bem como à postura adotada no momento da alimentação. Assim, as borboletas, as quais apresentam atividade diurna, guiam-se visualmente até uma dada flor e pousam nela quando se alimentam. As mariposas, de hábito noturno, ao contrário, guiam-se principalmente pelo olfato e normalmente não costumam pousar na flor enquanto se alimentam (FAEGRI \& PIJL, 1971; BARTH, 1991).

As borboletas, bem como a grande maioria dos lepidópteros, alimentam-se do néctar das flores de angiospermas. No gênero Heliconius Kluk, 1802 (Nymphalidae), estão reunidas espécies que utilizam regularmente também o pólen das flores como alimento. GiLBERT (1972) descreveu o elaborado comportamento relacionado ao processamento do pólen coletado pelos heliconíneos, o qual inclui provavelmente a mistura de pólen seco ora coletado com o néctar regurgitado, previamente ingerido. Ao contrário do proposto por este autor, KRENN \& PENZ (1998) sugeriram entretanto que, embora haja características exclusivas na probóscide das espécies que utilizam o pólen, estas não se restringem a Heliconius, mas, sim, ocorrem de modo similar nos demais ninfalídeos. Os nutrientes provenientes do pólen são de importância na manutenção do adulto. Relacionam-se diretamente à alta longevidade, bem como à taxa de oviposição constante, embora baixa, ao longo de toda a vida (GILBERT, 1972; DunLAP-PIANKa et al., 1977). Tanto o pólen ingerido pelas fêmeas, quanto os nutrientes que provêm daquele consumido pelos machos, contribuem para a produção de ovos. No caso dos machos, esses nutrientes são transferidos para a fêmea provavelmente por meio do espermatóforo (BOGGS \& GILBERT, 1979).

Gilbert (1975) discorreu sobre a associação Heliconius e dois gêneros de cucurbitáceas na Costa Rica, Anguria L. e Gurania Cogn., que são utilizados regularmente e polinizados por essas borboletas. A verbenácea Lantana camara L. é também destacada como fonte usual de néctar e pólen (GILBERT, 1972). Esta é a espécie mais visitada por Heliconius erato phyllis (Fabricius, 1775) em três áreas de São Paulo (RAmos \& Freitas, 1999). Romanowski et al. (1985), Ruszczyk (1986) e Moreira et al. (1996) tratam dos recursos alimentares utilizados pelos adultos de H. erato phyllis no Rio Grande do Sul. Os dois primeiros registraram as espécies utilizadas por $H$. erato phyllis e o último, a associação entre essa borboleta e a orquidácea Habenaria pleiophylla Hoenhe \& Schlechter. Desconhecemos, entretanto, qual o padrão floral destas plantas, se existente.

Objetiva-se caracterizar as flores usadas por H. erato phyllis no Rio Grande do Sul, em quatro áreas fisionomicamente distintas, associando à análise morfométrica da probóscide dos adultos e das estruturas florais onde o néctar é acumulado na flor. 


\section{MATERIAL E MÉTODOS}

Áreas de estudo. As quatro áreas escolhidas caracterizam desde ambientes relativamente preservados, Parque Estadual de Itapuã e Estação Experimental de Maquiné, a muito alterados, Jardim Botânico e Horto Florestal Barba Negra.

O Parque Estadual de Itapuã localiza-se no município de Viamão (30²3' S e 515ำ W). É formado por uma densa vegetação secundária, em grande parte composta por mirtáceas. Dentre as espécies encontradas situamse: Actinostemon concolor (Spreng.) Muell. Arg. (Euphorbiaceae), Casearia sylvestris Sw. (Flacourtiaceae), Piper gaudichaudianum Kunth (Piperaceae) e Ruellia angustiflora (Nees) Lindau ex Rambo (Acanthaceae). A Estação Experimental de Maquiné está situada na Mata Atlântica e dista $138 \mathrm{~km}$ de Porto Alegre $\left(29^{\circ} 39^{\prime} \mathrm{S}\right.$ e $50^{\circ} 12^{\prime}$ W). Na mata secundária da Estação, dentre as espécies do estrato arbustivo, há $P$. gaudichaudianum, Psychotria brachyceras Muell. Arg. (Rubiaceae) e Triumfetta semitriloba L. (Tiliaceae). O Jardim Botânico, situado em Porto Alegre (30 $02^{\prime}$ S e $\left.51^{0} 13^{\prime} \mathrm{W}\right)$, apresentava-se na ocasião com predomínio de espécies ornamentais cultivadas. Dentre estas destacaram-se, em abundância, Dahlia pinnata Voss e Zinnia elegans Jacq. (Asteraceae), Impatiens walleriana Hook. (Balsaminaceae) e Stachytarpheta cayennensis (Rich.) Vahl (Verbenaceae). O Horto Florestal da Barba Negra situa-se em Barra do Ribeiro ( $30^{\circ} 23^{\prime} \mathrm{S}$ e $51^{\circ} 12^{\prime} \mathrm{W}$ ). A maior parte deste é constituída de uma mata composta por diversas espécies de Eucalyptus L'Herit. acompanhada de um denso sub-bosque, composto predominantemente por espécies nativas. Dentre estas destacam-se o butiá, Butia capitata (Martius) Beccari (Arecaceae) e a capororoca, Myrsine umbellata Mart. (Myrsinaceae); ainda, Rumohra adiantiformis (Forst.) Ching (Dryopteridaceae), Desmodium sp. (Fabaceae), Stachytarpheta cayennensis (Rich.) Vahl e Melothria cucumis Cogn. (Cucurbitaceae).

Durante os meses de dezembro de 1996 a maio de 1997, cada uma das áreas foi amostrada em intervalos de aproximadamente 30 dias, totalizando seis dias de observação por área, num período amostral diário de cerca de três horas, a partir de $8 \mathrm{~h} 30 \mathrm{~min}$. Em cada local, foi previamente demarcada uma trilha, com cerca de $200 \mathrm{~m}$ de extensão e aproximadamente $4 \mathrm{~m}$ de largura, que correspondeu à área amostral.

Durante as amostragens, os adultos de H. erato phyllis foram observados quanto à atividade alimentar, adotando-se como sinal afirmativo de alimentação o pouso em uma dada flor e inserção da probóscide no tubo floral. Neste caso, o adulto era capturado com rede entomológica, identificado com base nos critérios descritos em HOLZINGER \& HolZINGER (1994), numerado com caneta de retroprojeção para evitar sua reutilização e, então, liberado. Em laboratório, a partir de uma população adicional do Horto Florestal Barba Negra $(n=20)$, mediu-se a probóscide dos adultos em relação à largura e ao comprimento.

As plantas utilizadas pelo inseto recebiam etiquetas numeradas em campo, eram fotografadas, e delas eram coletados ramos com flores para herborização, identificação e conservação em álcool 50\%. Depósitos correspondentes foram feitos no herbário ICN, do Departamento de Botânica/IB, da Universidade Federal do Rio Grande do Sul, sob números 119219 a 119242. Com o auxílio de um estereomicroscópio equipado com escala micrométrica ou paquímetro, mediu-se, de cada flor ( $\mathrm{n}=10 /$ espécie), a largura e o comprimento da estrutura armazenadora de néctar (tubo, câmara ou calcar). Sob estereomicroscópio equipado com grade ocular, as flores foram desenhadas esquematicamente em vista lateral externa e interna (corte sagital).

\section{RESULTADOS E DISCUSSÃO}

Registrou-se a alimentação de $H$. erato phyllis em 23 espécies vegetais (tab. I), pertencentes a 11 famílias, as quais variaram dentre os locais, conforme a disponibilidade correspondente. As plantas distribuem-se entre diversos hábitos, encontrando-se tanto ervas quanto lianas, epífitos, arbustos e árvores, predominando as primeiras. Quanto ao padrão de distribuição das flores na planta, se em inflorescências ou isoladamente, predominaram as inflorescências. Houve ampla variação relativa às cores das flores utilizadas (tab. I). Quanto à forma, as flores utilizadas podem ser categorizadas em três tipos: 1, tubiforme (figs. 1-14, 19-38); 2, calcarada (figs. 15, 16, 39, 42) e 3, provida de câmara para acúmulo de néctar (figs. 17, 18, 40, 41). Predominam as do primeiro tipo floral, tendo como exemplo Lantana camara (figs. 5, 20) e Stachytarpheta cayennensis (figs. 6, 24). O restante das flores divide-se igualmente entre os tipos 2 e 3 (tab. I); exemplos destes são, respectivamente, Impatiens walleriana Hook. (Balsaminaceae) 
Tabela I. Características das espécies vegetais cujas flores foram utilizadas por adultos de Heliconius erato phyllis em atividade de alimentação e o respectivo número de indivíduos observados por local ( A, Horto Florestal Barba Negra; B, Parque Estadual de Itapuã; C, Estação Experimental de Maquiné; D, Jardim Botânico, RS, de dezembro 1996 a maio de 1997 (in, inflorescência; is, flor isolada); cor predominante, segundo VILLALobos - Dominguez \& Villalobos (1947): B, branco; G, verde; L, verde-limão; M, magenta; O, laranja; R, rubi; S, escarlate; Y, amarelo; 1 , tubiforme; 2, calcarada; 3 , com câmara para acúmulo de néctar; dimensões referem-se à estrutura armazenadora de néctar $(\mathrm{n}=10$ flores, por espécie).

\begin{tabular}{|c|c|c|c|c|c|c|c|c|c|c|c|}
\hline \multirow{3}{*}{ Espécie } & \multirow{3}{*}{ Hábito } & \multicolumn{5}{|c|}{ Flores } & & & & & \multirow[b]{3}{*}{ Total } \\
\hline & & \multicolumn{2}{|c|}{ Padrão Cor } & \multirow[t]{2}{*}{ Tipo } & \multicolumn{2}{|c|}{ Dimensão ( mm; $\bar{x} \pm E P)$} & \multicolumn{4}{|c|}{ Adultos } & \\
\hline & & & & & Largura & Comprimento & A & $\mathrm{B}$ & $\mathrm{C}$ & $\mathrm{D}$ & \\
\hline Asclepias curassavica & erva & in & OY & 3 & $1,47+0,03$ & $3,26+0,06$ & 1 & & & & 1 \\
\hline Borreria fastigiata & erva & in & MR & 1 & $0,83+0,05$ & $2,69+0,04$ & & & 2 & & 2 \\
\hline Calliandra tweedii & arbusto & in & RS & 1 & $1,88+0,24$ & $7,05+0,13$ & & & & 1 & 1 \\
\hline Chomelia obtusa & árvore & is & SO & 1 & $1,03+0,07$ & $13,55+0,22$ & & 3 & & & 3 \\
\hline Cordia verbenacea & erva & is & MR & 1 & $2,22+0,14$ & $5,10+0,16$ & 1 & & & & 1 \\
\hline Dahlia pinnata & erva & is & $\mathrm{RS}$ & 1 & $1,13+0,06$ & $8,55+0,29$ & & & & 18 & 18 \\
\hline Dicliptera tweediana & erva & is & SO & 1 & $2,93+0,20$ & $28,90+0,70$ & & & 1 & & 1 \\
\hline Habenaria pleiophylla & erva & in & LLG & 2 & $0,83+0,06$ & $20,38+0,26$ & & 1 & & & 1 \\
\hline Impatiens walleriana & erva & is & M & 2 & $0,75+0,07$ & $26,45+1,25$ & & & & 1 & 1 \\
\hline Lantana camara & erva & in & OY & 1 & $1,00+0,04$ & $9,75+0,18$ & 2 & 3 & & 1 & 6 \\
\hline Manettia inflata & liana & is & SSO & 1 & $2,83+0,09$ & $12,60+0,36$ & & & 1 & & 1 \\
\hline Maranta divaricata & erva & is & B & 1 & $2,86+0,19$ & $16,25+0,33$ & 4 & & & 2 & 6 \\
\hline M. leuconeura & erva & is & B & 1 & $1,61+0,04$ & $8,35+0,36$ & & & & 2 & 2 \\
\hline Melothria cucumis & liana & is & OY & 1 & $1,48+0,06$ & $2,36+0,08$ & 1 & & & & 1 \\
\hline Oxypetalum parvifolium & liana & in & B & 3 & $0,69+0,03$ & $2,73+0,03$ & 1 & & & & 1 \\
\hline Richardia brasiliensis & erva & in & $\mathrm{B}$ & 1 & $1,19+0,10$ & $2,91+0,11$ & & & & 1 & 1 \\
\hline Solidago chilensis & erva & in & YYO & 1 & $0,44+0,04$ & $3,94+0,08$ & & & 1 & & 1 \\
\hline Stachytarpheta cayennensis & erva & in & MR & 1 & $1,15+0,06$ & $7,38+0,59$ & 11 & 2 & & 10 & 23 \\
\hline Tillandsia geminiflora & epífita & in & MMR & 1 & $2,50+0,11$ & $19,62+0,30$ & & 1 & & & 1 \\
\hline Tithonia diversifolia & erva & in & OOS & 1 & $1,16+0,04$ & $8,30+0,17$ & & & & 2 & 2 \\
\hline Triumfetta abutiloides & erva & in & YYO & 1 & $2,19+0,07$ & $8,60+0,14$ & 1 & & & & 1 \\
\hline Vernonia tweediana & erva & in & MR & 1 & $0,82+0,08$ & $7,95+0,20$ & & & 2 & & 2 \\
\hline Zinnia elegans & erva & in & M & 1 & $0,74+0,02$ & $5,97+0,21$ & & & & 5 & 5 \\
\hline
\end{tabular}

(figs. 16, 39) e Asclepias curassavica L. (Asclepiadaceae) (figs. 17, 41).

Acrescentam-se 17 citações novas de espécies de plantas ao total de 39 angiospermas já conhecidas nominalmente como fonte de alimentação para os adultos de H. erato phyllis no Brasil (tab. II). Os dados indicam que a abundância relativa de uma dada espécie de planta condiciona em grande parte o seu uso em cada um dos locais considerados. S. cayennensis e L. camara foram largamente utilizadas nos locais estudados, exceto na Estação Experimental de Maquiné, onde estas espécies não foram observadas na área amostral. Os resultados obtidos corroboram aqueles de RomANOwSKI et al. (1985) e de Ramos \& Freitas (1999), que apontam para a existência de uma grande plasticidade ecológica quanto à variação dos recursos alimentares utilizados pelos adultos de $H$. erato phyllis, à qual respondem rapidamente tanto em escala temporal quanto espacial.

Quanto as dimensões dos tubos florais (tab. I), os comprimentos médios mínimo e máximo foram encontrados, respectivamente, em Melothria cucumis e Dicliptera tweediana Nees (Acanthaceae). As larguras e as médias mínima e máxima foram observadas, respectivamente, em Solidago chilensis Meyen (Asteraceae) e D. tweediana. A média para o total de tubos florais do tipo 1 (tubiforme) foi de $10,50 \mathrm{~mm}$, para o comprimento, e de $2,23 \mathrm{~mm}$, para a largura. $\mathrm{O}$ comprimento médio encontrado para a 

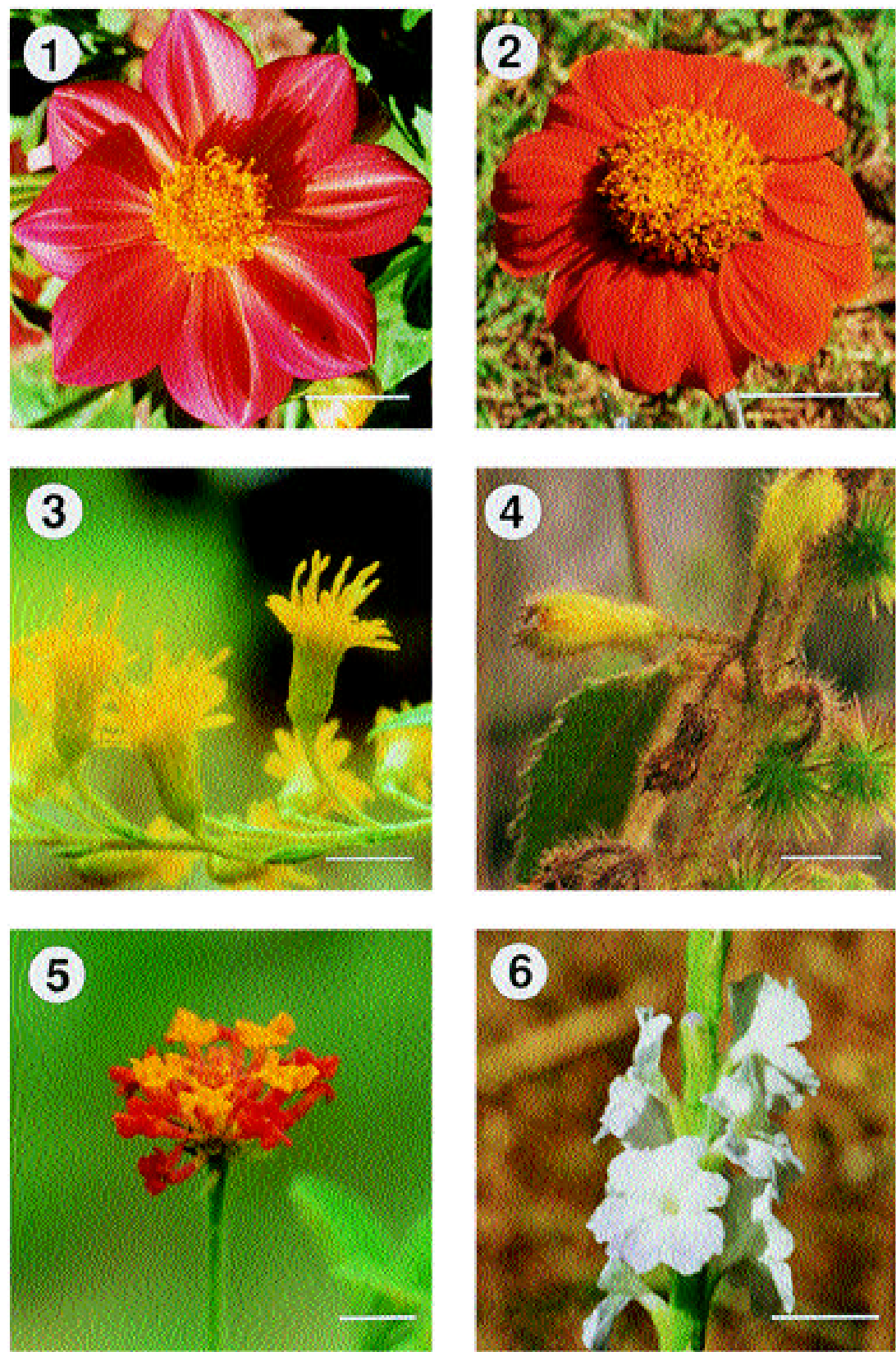

Figs. 1-6. Flores ou inflorescências de plantas utilizadas por Heliconius erato phyllis: 1, Dahlia pinnata; 2, Tithonia diversifolia; 3, Solidago chilensis; 4, Triumfetta abutiloides; 5, Lantana camara; 6, Stachytarpheta cayennensis. Escalas: 3mm, fig.3; 7mm, fig.4; 10mm, fig.5, 6; 15mm, figs.1, 2. 
Tabela II. Relação das plantas cujas flores são utilizadas por Heliconius erato phyllis no Brasil. 1, presente estudo; 2, Romanowski et al. (1985); 3, RuszczyK (1986); 4, Moreira et al. (1996); 5, Ramos \& Freitas (1999).

\begin{tabular}{|c|c|c|}
\hline Família & Espécie & Fonte \\
\hline \multirow[t]{3}{*}{ Acanthaceae } & Dicliptera tweediana Nees & 1 \\
\hline & Justicia brasiliana Roth & 3 \\
\hline & Ruellia sp. & 2 \\
\hline \multirow[t]{2}{*}{ Asclepiadaceae } & Asclepias curassavica $\mathrm{L}$. & 1,5 \\
\hline & Oxypetalum parvifolium Phil. & 1 \\
\hline \multirow[t]{21}{*}{ Asteraceae } & Aspilia buphtalmiflora (DC.) Bak. & 2 \\
\hline & Baccharis articulata (DC.) Bak. & 2 \\
\hline & B. genistelloides Bak. & 2 \\
\hline & Baccharis sp. & 2 \\
\hline & Bidens pilosa $\mathrm{L}$. & 2,5 \\
\hline & Dahlia pinnata Voss & 1 \\
\hline & Elephantopus sp. & 2 \\
\hline & Emilia sonchifolia DC. & 5 \\
\hline & Eupatorium laevigatum DC. & 5 \\
\hline & Eupatorium sp. & 2 \\
\hline & Erechthites sp. & 2 \\
\hline & Mikania lundiana DC. & 5 \\
\hline & Mikania sp. & 2 \\
\hline & Senecio brasiliensis Bak. & 2 \\
\hline & Solidago chilensis Meyen & 1 \\
\hline & Tithonia diversifolia Blake & 1 \\
\hline & T. speciosa Hook. ex Gris. & 5 \\
\hline & Trixis antimenorrhoea Mart. ex Baker & 5 \\
\hline & Vernonia tweediana Bak. & 1,2 \\
\hline & Vernonia sp. & $2,3,5$ \\
\hline & Zinnia elegans Jacq. & 1 \\
\hline Balsaminaceae & Impatiens walleriana Hook. & 1,5 \\
\hline Boraginaceae & Cordia verbenacea DC. & 1 \\
\hline \multirow[t]{2}{*}{ Bromeliaceae } & Tillandsia aeranthos (Loisel.) L.B. Smith & 2 \\
\hline & T. geminiflora $\mathrm{Mez}$ & 1 \\
\hline Cactaceae & Rhipsalis sp. & 2 \\
\hline \multirow{2}{*}{ Cucurbitaceae } & Melothria cucumis Cogn. & 1 \\
\hline & Gurania sp. & 5 \\
\hline Fabaceae & Calliandra tweedii Benth. & 1,2 \\
\hline Lythraceae & Heimia salicifolia Link \& Otto & 2 \\
\hline Malpighiaceae & Janusia guaranitica (St. Hil.) Juss. & 2 \\
\hline Malvaceae & Sida rhombifolia L. & 2 \\
\hline \multirow[t]{2}{*}{ Marantaceae } & Maranta divaricata Rosc. & 1 \\
\hline & M. leuconeura Peters & 1 \\
\hline Myrtaceae & Eugenia uniflora $\mathrm{L}$. & 2 \\
\hline Nyctaginaceae & Bouganvillea glabra Choisy & 3 \\
\hline \multirow[t]{2}{*}{ Orchidaceae } & Habenaria pleiophylla Hoehne \& Schlechter & 1,4 \\
\hline & Epidendrum fulgens Brongn. & 5 \\
\hline Oxalidaceae & Oxalis sp. & 2 \\
\hline Rosaceae & Rubus rosifolius Smith & 5 \\
\hline \multirow[t]{5}{*}{ Rubiaceae } & Borreria fastigiata $\mathrm{K}$. Sch. & 1 \\
\hline & Chomelia obtusa C. \& S. & 1 \\
\hline & Manettia inflata Sprague & 1 \\
\hline & Richardia brasiliensis Gomes & 1 \\
\hline & Richardia sp. & 2 \\
\hline \multirow[t]{2}{*}{ Solanaceae } & Acnistus breviflorus Sendtner & 2 \\
\hline & Solanum sp. & 2 \\
\hline Tiliaceae & Triumfetta abutiloides St. Hil. & 1 \\
\hline \multirow[t]{3}{*}{ Verbenaceae } & Lantana camara $\mathrm{L}$. & $1,3,5$ \\
\hline & Stachytarpheta cayennensis (Rich.) Vahl & 1 \\
\hline & S. polyura L. & 5 \\
\hline
\end{tabular}



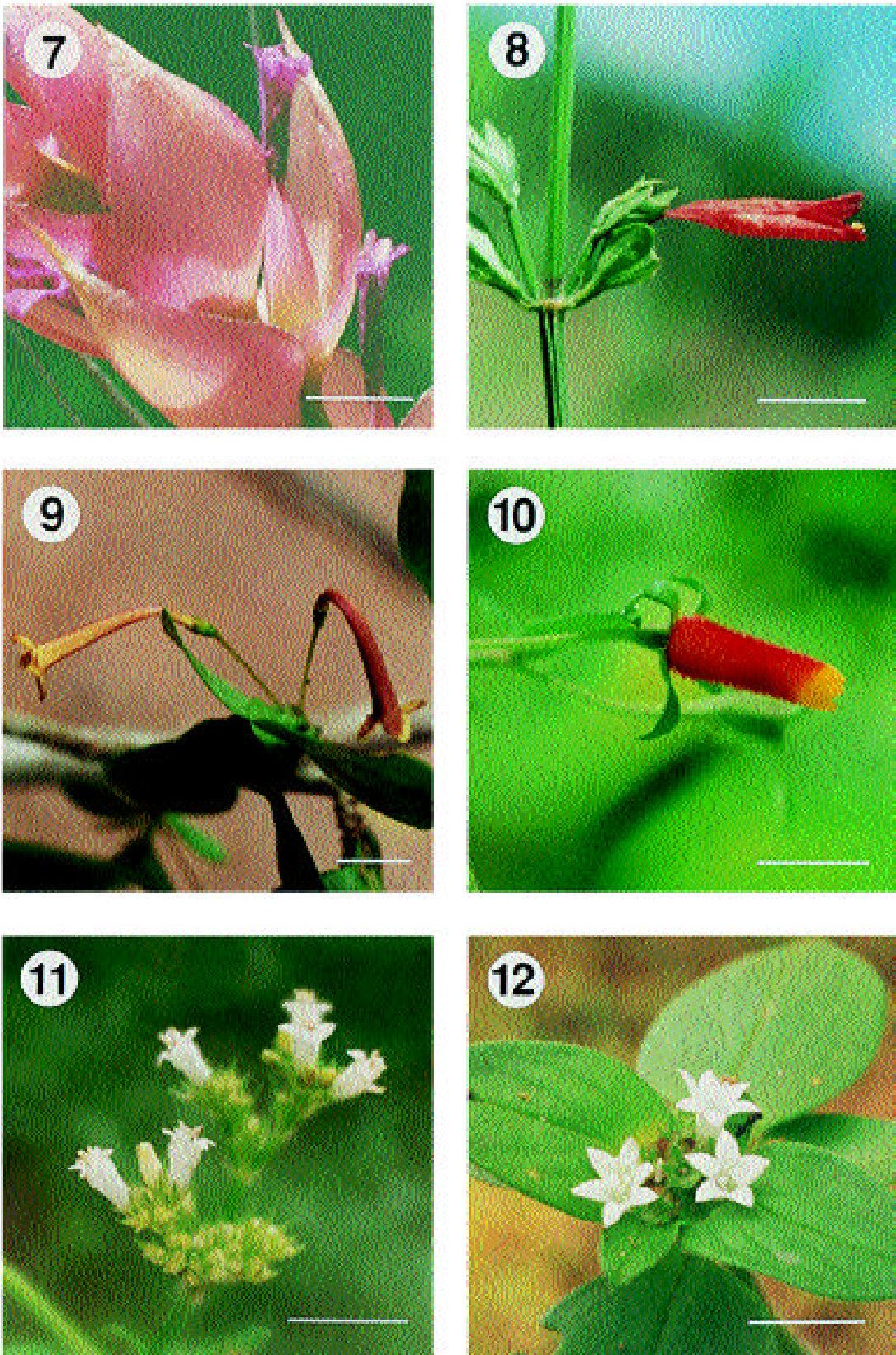

Figs. 7-12. 7, Tillandsia geminiflora; 8, Dicliptera tweediana; 9, Chomelia obtusa; 10, Manettia inflata; 11, Borreria fastigiata; 12, Richardia brasiliensis. Escalas: 5mm, figs. 9, 11; 7mm, figs. 7, 12; 8mm, fig. 10;15mm, fig. 8 . 
probóscide de $H$. erato phyllis foi de $18,01 \mathrm{~mm}$, e a média da largura máxima correspondente, de $0,39 \mathrm{~mm}$.

Não há indicação de que a variação, quanto às características das flores utilizadas, difira substancialmente daquela observada nas demais espécies já assinaladas na literatura. A associação predominante com flores do tipo tubiforme sugere que o caso de $H$. erato phyllis enquadrar-se-ia numa síndrome floral típica de psicofilia (sensu FAEGRI \& PIJL, 1971), mesmo levando-se em consideração as já conhecidas limitações deste enfoque (BERTIN, 1989). O aspecto tubiforme e a dimensão das flores utilizadas, pequenas em sua grande maioria, vão ao encontro da morfologia do aparelho bucal do adulto, ou seja, a existência de uma relação direta entre o tamanho do tubo floral e o da probóscide. As flores utilizadas apresentam tubos florais de dimensões em média menores em comprimento e maiores em largura, que aquelas da probóscide de $H$. erato phyllis. As únicas exceções correspondem a $D$. tweediana (tipo 1 ), $H$. pleiophylla e $I$. walleriana (tipo 2), quanto ao comprimento. A primeira apresenta a corola gamopétala até aproximadamente a metade do tubo e a porção distal livre. Esta última é distendida lateralmente, tornando-se suficientemente larga, ao ponto de permitir até mesmo a inserção da cabeça de $H$. erato phyllis na flor, por ocasião da alimentação, conforme diversas observações por nós realizadas. Aquelas do tipo 2 apresentam, na maioria das vezes, o calcar semi- ou totalmente preenchido com néctar, o que permite a ingestão deste sem atingir necessariamente a extremidade do calcar com a probóscide.

Tais aspectos justificariam a existência de uma convergência morfométrica entre essas duas estruturas, o que precisa ser avaliado, em caso da confirmação de $H$. erato phyllis como um polinizador efetivo das angiospermas citadas. A existência de correlação entre as dimensões do tubo floral de uma dada espécie e seu agente polinizador tem sido comumente apontada como evidência indireta da coevolução destas duas estruturas (Herrera, 1996). Esta provável coevolução inseto-planta remonta ao Cretáceo, período em que os tubos florais e as probóscides dos insetos alongaram-se paralelamente (TAKHTAJAN, 1991). Há indicações recentes de que nas populações naturais de algumas variedades de flores e de espécies de borboletas tais caracteres são concomitantemente modificados ao longo do tempo (SHIELDS, 1972).

A dúvida com relação ao desempenho das borboletas e mariposas como agentes polinizadores efetivos diz respeito ao critério de fidelidade (SHIELDS, 1972; CREPET, 1983), visto que geralmente visitam diversas angiospermas, o que é também válido para $H$. erato phyllis. Dentro do contexto da polinização, entretanto, Heliconius diferencia-se por agrupar espécies que realizam coleta de pólen. H. erato (Linnaeus, 1758), por exemplo, retira o pólen de $L$. camara de forma ativa, levando mais tempo para extraí-lo de suas flores quando comparado com a coleta de néctar desta verbenácea (GILBERT, 1972). Estudos conduzidos na Costa Rica comprovaram a retirada de pólen e respectiva importância quanto à polinização, de cucurbitáceas dos gêneros Anguria e Gurania por H. erato (GILBERT, 1975, 1991). Adicionalmente, foi demonstrado em campo que Heliconius ethilla Godart, 1819 retira cargas de pólen da rubiácea Palicourea crocea Schlechter (GILBERT, 1972). Tal comportamento alimentar aumenta o potencial das espécies de Heliconius como polinizadores efetivos, dada a formação de uma massa considerável de pólen na probóscide durante a coleta e possibilidade de tranferência desta para outras flores, em visitas subsequentes. O mecanismo de retirada do pólen por $H$. erato phyllis permanece desconhecido (KRENN \& PENZ, 1998). As presentes observações indicam que nas flores 

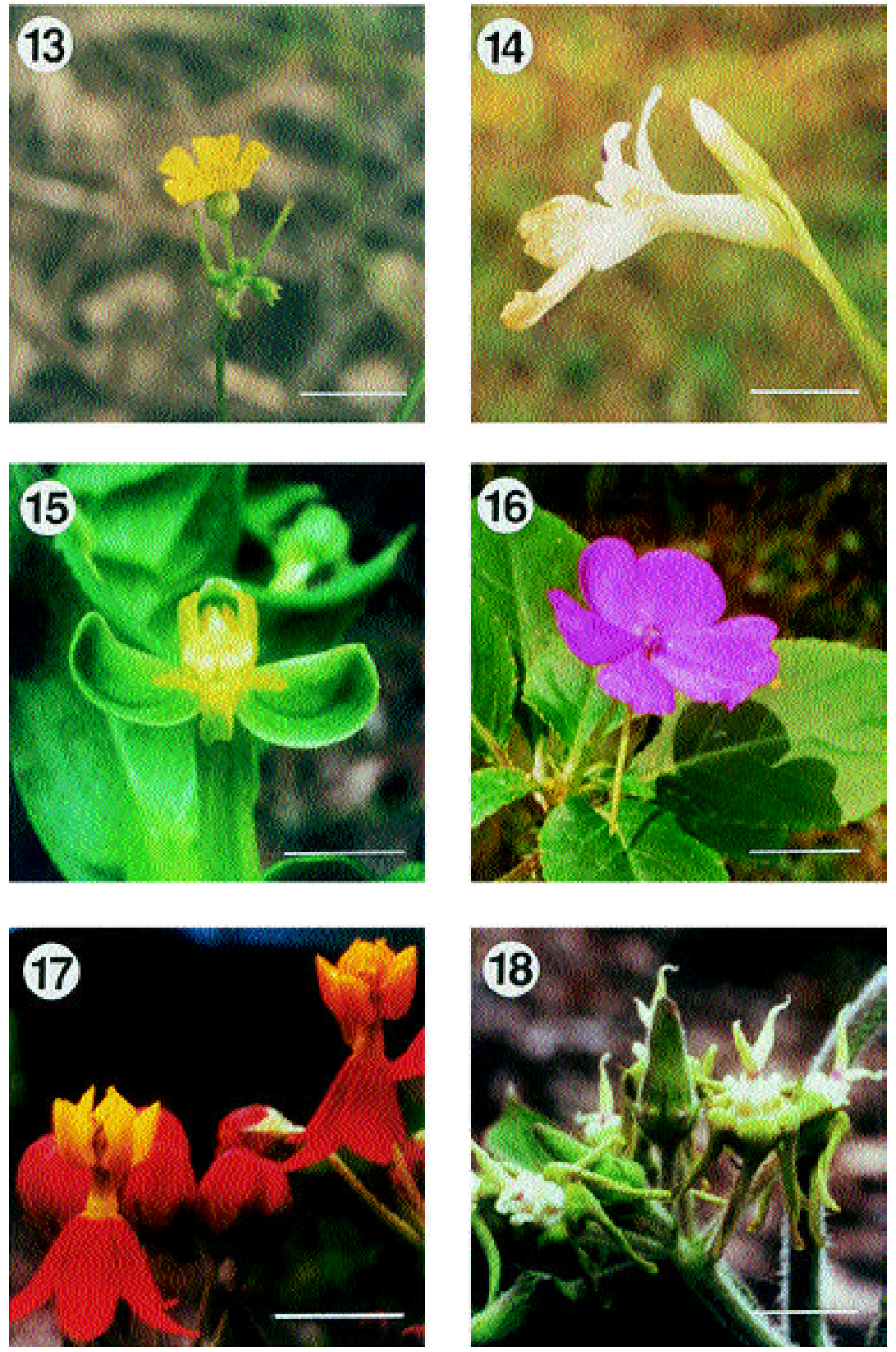

Figs. 13-18. 13, Melothria cucumis; 14, Maranta leuconeura; 15, Habenaria pleiophylla; 16, Impatiens walleriana; 17, Asclepias curassavica ; 18, Oxypetalum parvifolium. Escalas: 5mm, figs. 13, 14; 7mm, figs. 15 , $18 ; 10 \mathrm{~mm}$, fig. $17 ; 15 \mathrm{~mm}$, fig. 16 . 


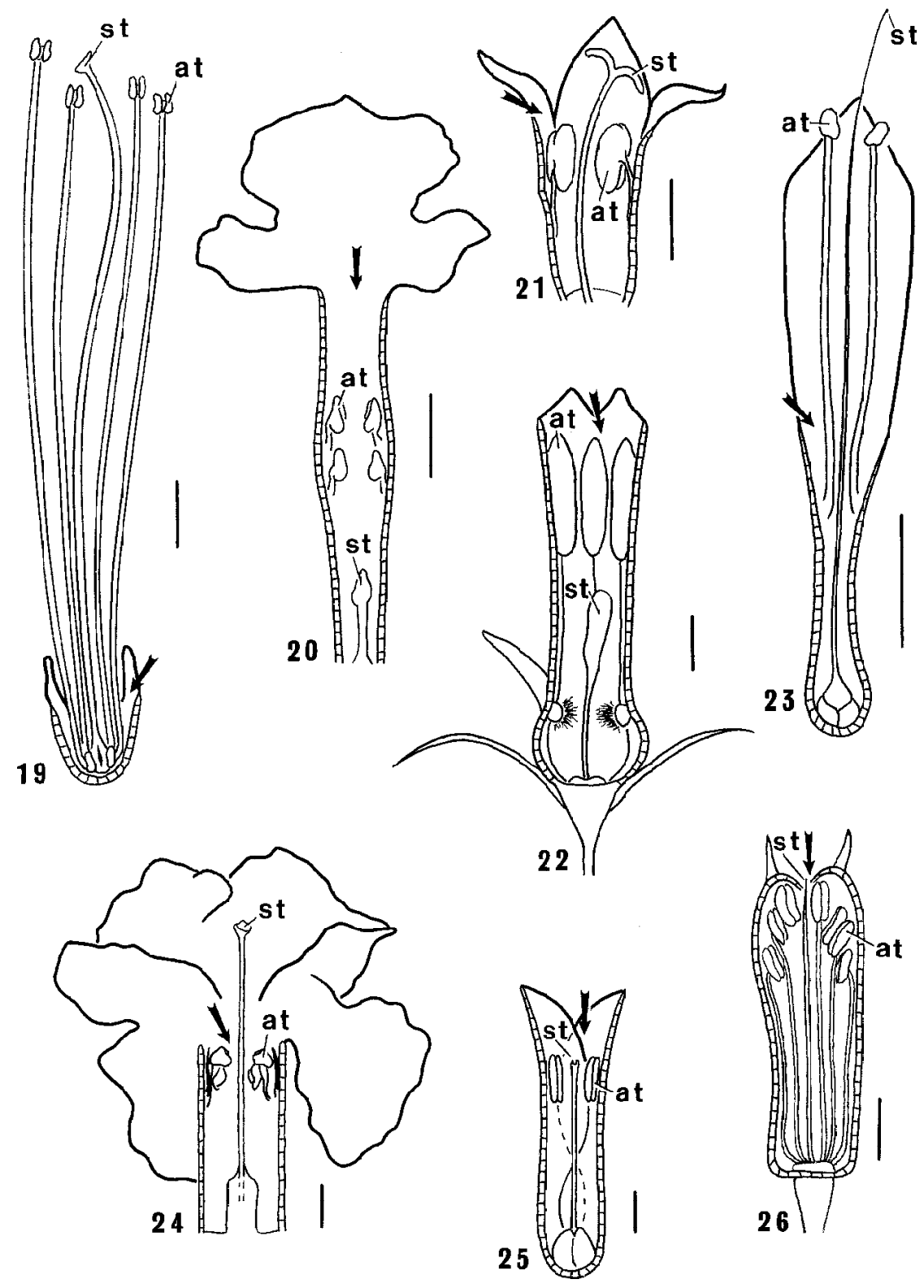

Figs. 19-26. Representação esquemática da morfologia interna das flores utilizadas por Heliconius erato phyllis, em corte sagital. 19, Calliandra tweedii; 20, Lantana camara; 21, Borreria fastigiata; 22, Manettia inflata; 23, Dicliptera tweediana; 24, Stachytarpheta cayennensis; 25, Tillandsia geminiflora; 26, Triumfetta abutiloides. Setas indicam local de inserção da probóscide. (at, antera; st, estigma). Escalas: 1mm, figs.21, 24; 1,5mm, figs. 19, 26; 2,5mm, figs. 20, 22; 4mm, fig. 25; 6mm, fig. 23 . 


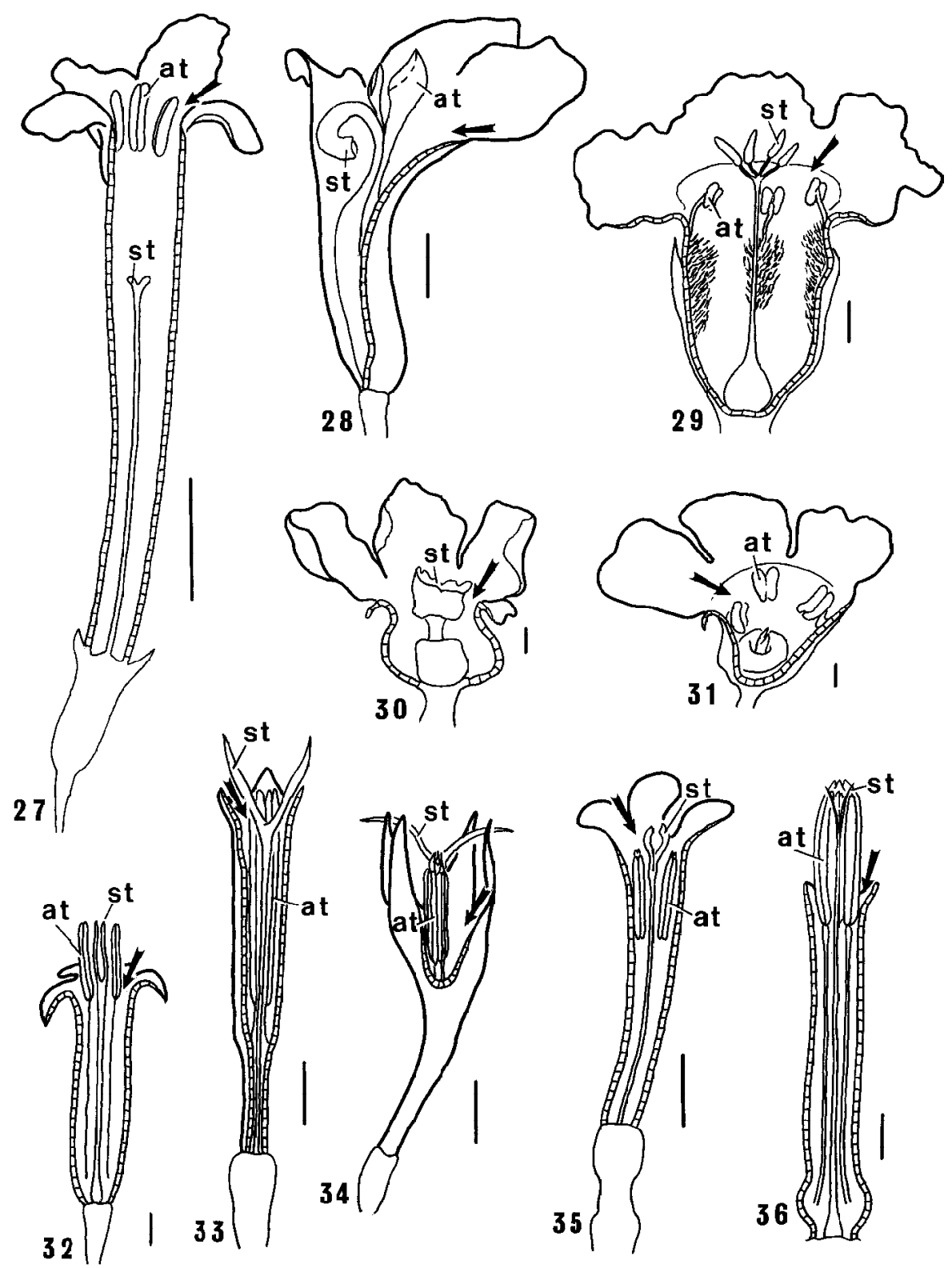

Figs. 27-36. 27, Chomelia obtusa; 28, Maranta leuconeura; 29, Cordia verbenacea; 30, Melothria cucumis, feminina; 31, Melothria cucumis, masculina; 32, Solidago chilensis; 33, Dahlia pinnata; 34, Vernonia tweediana; 35, Zinnia elegans; 36, Tithonia diversifolia (at, antera; st, estigma). Escalas: 0,5mm, figs. 30-32; 1mm, fig. 29; 1,5mm, figs. 34, 36; 2mm, figs. 28, 33, 35; 3mm, fig. 27. 

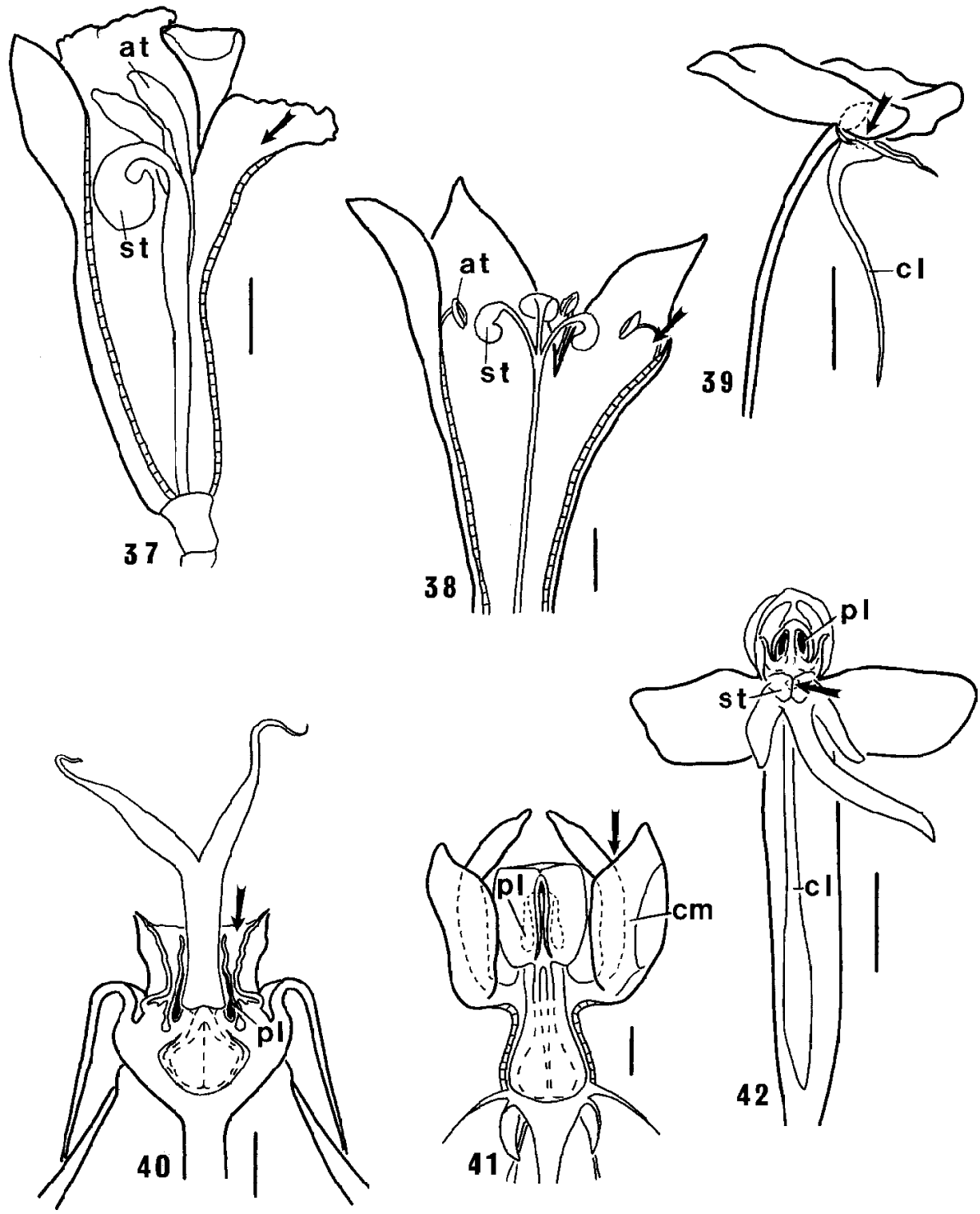

Figs. 37-42. 37, Maranta divaricata; 38, Richardia brasiliensis; 39, Impatiens walleriana; 40, Oxypetalum parvifolium; 41, Asclepias curassavica; 42, Habenaria pleiophylla. (at, antera; $\mathrm{cl}$, calcar; $\mathrm{cm}$, local de acúmulo de néctar; pl, polínia; st, estigma). Escalas: $1 \mathrm{~mm}$, figs. 38,$41 ; 1,5 \mathrm{~mm}$, fig. $40 ; 2,5 \mathrm{~mm}$, fig. $37 ; 5 \mathrm{~mm}$, fig. $42 ; 10$ mm, fig. 39 . 
onde este é retirado (L. camara e S. cayennensis), os verticilos florais (anteras e/ou estigmas) localizam-se na linha de inserção da probóscide (figs. 5, 6, 20 e 24). Tal fato, associado à reduzida largura dos tubos florais destas, sugere que tal mecanismo, se não em parte acidental, é grandemente beneficiado pelo arranjo correspondente.

A coleta ativa de uma quantidade substancial de pólen através da probóscide de H. erato phyllis, entretanto, não consta como único meio de transporte de pólen por este heliconíneo. A coleta passiva de pólen já foi observada em relação a Habenaria pleiophylla Hoehne \& Schlechter (Orchidaceae) (MoreIRA et al., 1996). Os adultos de $H$. erato phyllis, ao retirarem o néctar da orquidácea, ficam com polínias aderidas principalmente nos olhos e na cabeça. Observações de campo apontam para um transporte passivo de polínias de A. curassavica e Epidendrum fulgens Brongn. (Orchidaceae) aderidas à probóscide deste ninfalídeo (Ramos \& Freitas, 1999). Polínias de Asclepiadaceae (provavelmente A. curassavica) aderidas aos tarsos medianos e posteriores são comuns ao longo do ano para as populações de Maquiné e Horto Florestal Barba Negra. Dessa forma, a efetividade da polinização destas angiospermas por $H$. erato phyllis precisa também ser analisada.

Frente à diversidade de famílias, formas e cores das flores utilizadas, infere-se que $H$. erato phyllis comporta-se como espécie oportunista, ou seja, utiliza uma variedade considerável de flores disponíveis numa dada área, uma vez satisfeitos os padrões morfológicos passíveis de uso. A ampla variedade de cores das flores utilizadas sugere que não há uma cor preferencial. Muitas delas não apresentam cores intensas como, por exemplo, S. cayennensis (fig. 6), Richardia brasiliensis Gomes (Rubiaceae) (fig. 12) e H. pleiophylla (fig. 15), o que é característica das flores falenofílicas (FAEGRI \& PIJL, 1971). H. pleiophylla é, ainda, um exemplo de flor de atividade noturna, no que diz respeito à liberação de odor, outra característica de falenofilia. O uso desta espécie dá-se supostamente em situação de relativa escassez de alimento, prevalescente nas plantações de Eucalyptus do Horto Florestal Barba Negra (MoREIRA et al., 1996). Da mesma forma, o padrão de distribuição das flores na planta, se inflorescências ou flores isoladas, parece pouco importante como caráter seletivo. O discreto predomínio das inflorescências talvez justifique-se pelo maior apelo visual destas quando comparadas às flores isoladas (WEISS, 1991). Ainda, não parece haver restrições filogenéticas, uma vez que diversas famílias de plantas são utilizadas, desde aquelas tidas como relativamente ancestrais a derivadas. Desta forma, os resultados sugerem que o número de espécies de plantas utilizadas como recurso alimentar pelos adultos de H. erato phyllis aumentará significativamente à medida que outras áreas sejam estudadas a este respeito.

Os resultados obtidos têm implicações diretas quanto à manutenção de populações naturais de H. erato phyllis em áreas manejadas. À semelhança do obtido por GILBERT (1972) e DunLAP-PIANKA et al. (1977) para H. ethilla e Heliconius charitonius (Linnaeus, 1767) tanto o pólen quanto o néctar são limitantes para a sobrevivência e performance reprodutiva de $H$. erato phyllis. Conforme apontado por RuszCZYK (1986), os adultos deste ninfalídeo se alimentam preferentemente de flores umbrófilas, em matas relativamente conservadas. A predominância de espécies herbáceas utilizadas demonstra a respectiva importância deste estrato para o sucesso dos adultos, o qual é via de regra desprezado nas estratégias de manejo, sendo muitas vezes aparado em áreas residenciais, caminhos e até mesmo parques. O cultivo dessas espécies, ao contrário, deve ser fomentado, principalmente nos ambientes urbanos. 
Agradecimentos. Aos diretores do Jardim Botânico (FZB/RS), da Estação Experimental de Maquiné (FEPAGRO), do Parque Estadual de Itapuã (Secretaria da Agricultura) e do Horto Florestal Barba Negra (RIOCELL) por permitirem o uso das áreas de estudo. A Marcos Sobral (UFRGS) pelo auxílio na identificação das plantas e trabalho de campo, bem como pelas correções quanto à nomenclatura utilizada. A Nelson Matzenbacher (UFRGS), pelo auxílio na interpretação da morfologia floral em Asteraceae. Ao Marcos Sobral e Cláudio A. Mondin (UNISINOS) pela leitura crítica de versões preliminares do manuscrito. Parte dos custos deste estudo foi mantida pela FAPERGS, Projeto nº 94/50941.7, concedido a G.R.P. Moreira.

\section{REFERÊNCIAS BIBLIOGRÁFICAS}

BARTH, F. G. 1991. Insects and flowers. Princeton, Princeton University. 408 p.

Bertin, R. I. 1989. Pollination biology. In: ABRAHAMSON, W.G. ed. Plant-animal Interactions. New York, McGrawl-Hill. p.23-83.

Boggs, C. L. \& GILBERT, L. E. 1979. Male contribution to egg production in butterflies: evidence for transfer of nutrients at mating. Science, New York, 206: 83-84.

Crepet, W. L. 1983. The role of insect pollination in the evolution of the angiosperms. In: REAL, L. ed. Pollination Biology. Orlando, Academic. p. 29-50.

DARwIN, C. 1877. The various contrivances by which orchids are fertilised by insects. 2 ed., Chicago, University of Chicago. 300 p.

Dunlap-Pianka, H.; Boggs, C. L. \& Gilbert, L. E. 1977. Ovarian dynamics in heliconiine butterflies: programmed senescence versus eternal youth. Science, New York, 197: 487-490.

FAEGRI, K. \& PiJl, L. 1971. The principles of pollination ecology. 2 ed., Oxford, Pergamon. 291 p.

Gilbert, L. E. 1972. Pollen feeding and reproductive biology of Heliconius butterflies. Proc. natn. Acad. Sci. U.S.A, Washington, 69: 1403-1407.

1975. Ecological consequences of a coevolved mutualism between butterflies and plants. In: GILBERT, L.E. \& RAven, P.H. eds. Coevolution of animal and plants. Austin, University of Texas. p. 210- 240. 1991. Biodiversity of a Central American Heliconius community: pattern, process and problems. In: PRICE, P.; LEWINSOHN, T.M. et al. eds. Plant-animal interactions: evolutionary ecology in tropical and temperate regions. New York, John Wiley \& Sons. p. 403- 427.

HERRERA, C. 1996. Floral traits and plant adaptation to insect pollinators: a devil's advocate approach. In: LLOYD, D. G. \& BarRetT, C. H. eds. Floral biology: studies on floral evolution in animal pollinated plants. New York, Chapmann \& Hall. p. 65-87.

Holzinger, H. \& Holzinger, R. 1994. Heliconius and related genera. Venette, Sciences Nat. 328p.

KrenN, H. W. \& Penz, C. M. 1998. Mouthparts of Heliconius butterflies (Lepidoptera: Nymphalidae): a search for anatomical adaptations to pollen-feeding behavior. Int. J. Insect Morphol. \& Embryol., Kidlington, 27: 301309.

Moreira, G.R.P.; CorrêA, C. \& Mugrabi-Oliveira, E. 1996. Pollination of Habenaria pleiophylla Hoehne \& Schlechter (Orchidaceae) by Heliconius erato phyllis Fabricius (Lepidoptera; Nymphalidae). Revta bras. Zool., Curitiba, 13: 791-798.

Ramos, R. \& Freitas, A. V. 1999. Population biology and wing color variation in Heliconius erato phyllis (Nymphalidae). J. Lepid. Soc., Los Angeles, 53: 11-21.

Romanowski, H. P., Gus, R. \& Araúso, A. M. 1985. Studies on the genetics and ecology of H. erato (Lepid.; Nymph.) III. Population size, preadult mortality, adult resources and polymorphism in natural populations. Revta bras. Biol., Rio de Janeiro, 45: 563-569.

RuszCZYK, A. 1986. Hábitos alimentares de borboletas adultas e sua adaptabilidade ao meio urbano. Revta bras. Biol., Rio de Janeiro, 46: 419- 427.

ShIELDS, O. 1972. Flower visitation records for butterflies (Lepidoptera). Pan-Pac. Entomol., San Francisco, 48: 189-203.

TAKHTAJAN, A. 1991. Evolutionary trends in flowering plants. New York, Columbia University. 241 p.

Villalobos-Domiguez, C. \& Villalobos, J. 1947. Atlas de los colores. Buenos Aires, El Ateneo. 74p.

WeIss, M. R. 1991. Floral colour changes as cues for pollinators. Nature, London, 354: 227-229.

Recebido em 22.03.2000; aceito em 18.10.2000. 